\title{
Microporous Ceramic Membranes
}

\author{
H. P. Hsieh, ${ }^{* * 1}$ P. K. T. LiU, ${ }^{* 2}$ and T. R. Dillman ${ }^{* 2}$ \\ *1 Aluminum Co. of America, \\ Alcoa Laboratories, Alcoa Center, PA 15069, USA \\ *2 Aluminum Co. of America, \\ Alcoa Separations Technology, Inc., Warrendale, PA 15086, USA
}

(Received November 26, 1990)

\begin{abstract}
Microporous ceramic membranes are an emerging separation technology. Porous alumina, zirconia and glass membranes are discussed with emphasis on alumina membranes. The microstructural characteristics and element and module configurations of these membranes are examined in relation to their preparation methods -- anodic oxidation, phase separation/leaching and slipcasting. The membrane permeation properties are presented along with three applications: oil/water separation, protein separation and gas separation.

KEY WORDS: Alumina Membrane / Zirconia Membrane / Glass Membrane / Microstructure / Preparation Methods / Oil-Water Separation / Protein Separation / Gas Separation /
\end{abstract}

Organic polymeric membranes have enjoyed increasing success in process industries such as desalination, food and beverage, and waste water treatment. These membranes are typically cast by polymerization processes. In contrast to polymeric membranes, many ceramic membranes do not exhibit polymeric network and are made by starting with assemblies of crystals or particles.

As a result of the compact crystal structure, the chemical bonding and the high field strengths associated with the small and highly charged cations in ceramics, ceramic membranes are generally more stable chemically, structurally and thermally than organic membranes. Alumina and zirconia are two such examples. Although, strictly speaking, most of the glass membranes are not considered ceramic, their structures, properties and applications, however, are fairly similar to the other two membranes.

Alumina and zirconia membranes, particularly the former, are believed to derive from the uranium isotopes separation projects from the 1940s to the 1960s. Uranium enrichment for nuclear uses involves the separation of two isotopes: ${ }^{235} \mathrm{U}$ and ${ }^{238} \mathrm{U}$. Gas diffusion through membranes proved to be the most successful method for the isotope separation even though the membranes were often required to cascade up to more than a thousand stages. Alumina was selected for its corrosion resistance toward the gases involved.

In France the alumina membrane technology for gas diffusion applications went through some innovative evolutions, the most important of which was the modification and market development of alumina membranes for aqueous applications. It is believed that zirconia membranes went through a similar product development cycle in the U.S. and France. 
Contrary to alumina and zirconia membranes whose earlier developments have been confidential until recently, the microporous glass membranes have long been extensively studied in the open literature. Microporous glass membranes were marketed as a separation tool only recently.

\section{MEMBRANE CHARACTERISTICS}

Although some dense ceramic membranes exhibit excellent permselectivity, they are not attractive for industrial applications due to low permeabilities. Only porous membranes are discussed here. Commercial porous ceramic membranes show two major types of pore shape: nearly straight pores and tortuous pores (Figures 1 and 2).

Some commercial ceramic membranes have symmetric structures in which the general pore paths and sizes do not show distinctive differences across the thickness of the membrane elements. This type of symmetric structure (Figure 2), however, can impose significant pressure drops over a thick layer. To reduce the total hydraulic resistance through the membrane element, several membranes adopt a graded or composite structure in which the most selective layer (membrane) is kept as thin as possible and one or more layers of thicker support with larger pores are added to the membrane layer for mechanical strength and ease of permeate flow.

The nearly straight-pore membranes are currently limited to small laboratory applications. Furthermore, most of the ceramic membranes used on a large industrial scale are multilayered. Therefore, further discussions will be focused on the tortuous-pore composite membranes.

\section{$\underline{\text { Microstructure }}$}

The pore diameters of commercial ceramic membranes range from approximately 4 $\mathrm{nm}$ to $10 \mu \mathrm{m}$. Figure 3 shows an alumina membrane with a $0.2 \mu \mathrm{m}$ pore diameter superimposed on an intermediate layer of support and a bulk support. The resulting pore size distribution also reflects the asymmetric or multilayered structure.

\section{Permeation properties}

The use of graded pore sizes in composite layers to minimize pressure drop was demonstrated in the following experiment. A $2 \mathrm{~mm}$ thick symmetric alumina support disk with a uniform $0.2 \mu \mathrm{m}$ pore diameter had a water flux of $27000 \mathrm{~L} / \mathrm{m}^{2}-\mathrm{h}$ under $4 \mathrm{~atm}$. The disk was deposited with a thin layer $(5 \mu \mathrm{m})$ of boehmite which upon heating to $500^{\circ} \mathrm{C}$ was converted to transition alumina. The disk now containing the thin membrane layer and the bulk support was subsequently tested to show a water flux of $2500 \mathrm{~L} / \mathrm{m}^{2}-\mathrm{h}$. By assuming that the hydraulic resistance of the composite is equal to the sum of those of the membrane layer and the support, the water flux of the membrane layer can be calculated to be 2700 $\mathrm{L} / \mathrm{m}^{2}$-h. It is seen that the hydraulic resistance lies mostly in the membrane layer, when the membrane pore size is much smaller than that of the support.

One of the most important parameters in determining permeability is the membrane pore diameter. Shown in Figure 4 are the water permeabilities of alumina membrane monoliths with various pore diameters. The permeability varies as an exponential function of the pore diameter. It deviates from the simple Hagen-Poiseuille model which shows a quadratic dependence on the pore diameter. Most of the ceramic membranes have rather narrow pore size distributions, and, therefore, typically exhibit sharp retention curves, as shown in Figure 5. 


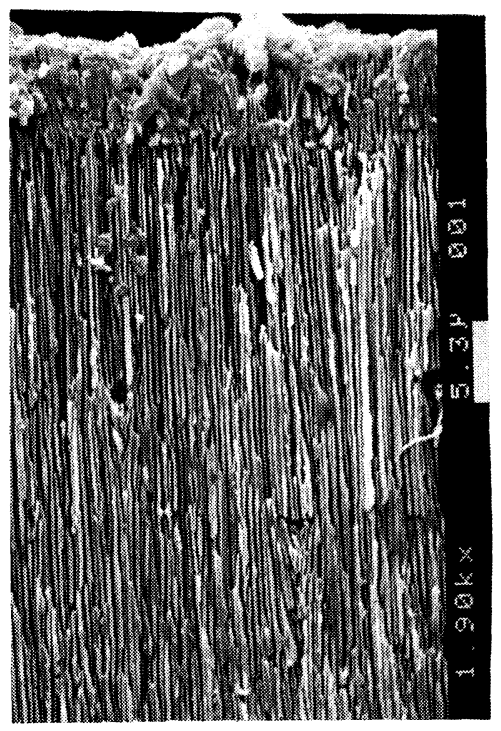

Figure 1. SEM of a nearly straight-pore alumina membrane.

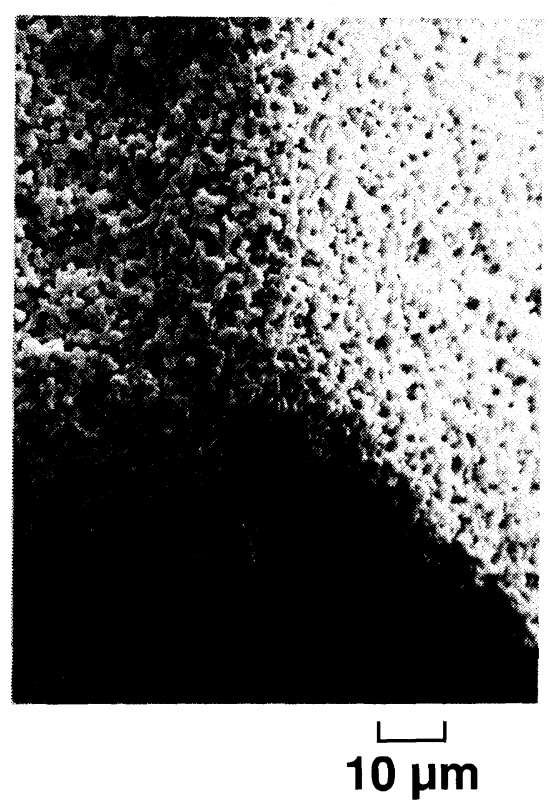

Figure 2. SEM of a symmetric, tortuous-pore glass membrane (courtesy of Asahi Glass).

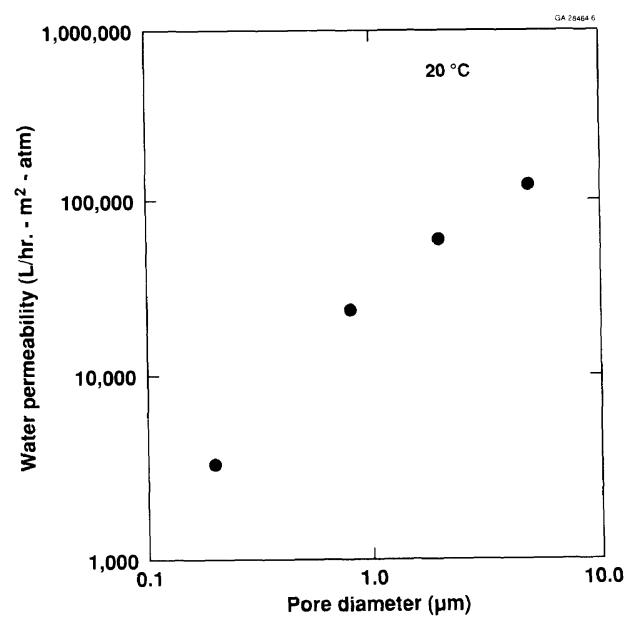

Figure 4. Pore size dependence of water permeability of alumina membranes. 


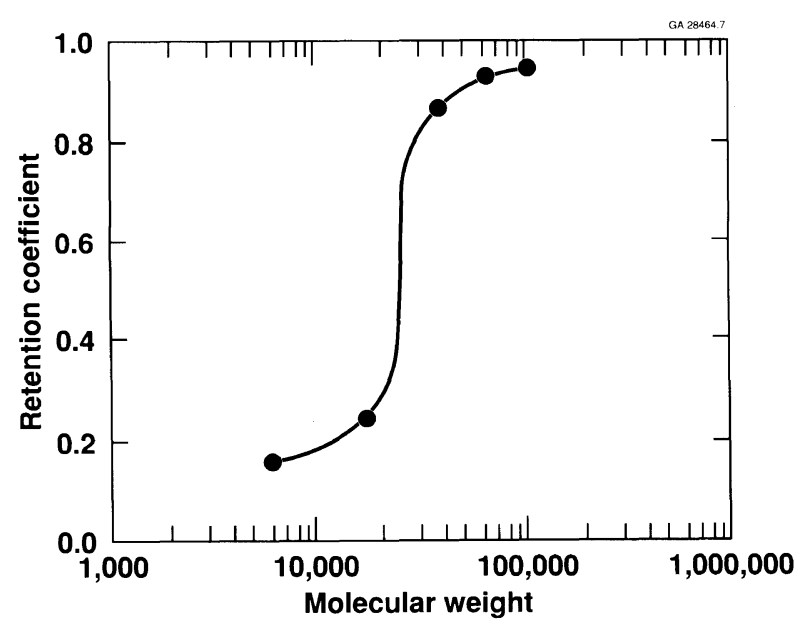

Figure 5. Retention curve of a $5 \mathrm{~nm}$ pore diameter zirconia membrane using dextrans as test molecules.

Similar to liquid permeation through microporous ceramic membranes, singlecomponent gas flux in most cases is found to be linearly proportional to the transmembrane pressure difference (TPD). Therefore, the gas permeability (gas flux/TPD) is, for practical purposes, independent of TPD. The transport mechanisms, however, appear to be more complex than liquid permeation and can vary depending on the membrane characteristics as well as the operating conditions. For example, laminar flow, turbulent flow, Knudsen diffusion, surface diffusion and capillary condensation or their combinations can govern the gas flow through microporous ceramic membranes.

\section{Flux regeneration}

One of the most important advantages of ceramic membranes is that, after fouling occurs, they are amenable to many flux regeneration schemes which are promoted by fluid dynamics, thermal or chemical treatment. In contrast, most organic polymeric membranes can only be subjected to rather mild regeneration methods. This difference, in many cases, is the primary consideration when selecting between ceramic and organic membranes.

Several flux regeneration methods for ceramic membranes have been used in various process industries and discussed elsewhere. ${ }^{1}$ Some examples include backflushing, fast flushing, flow reversal, chemical cleaning, steam sterilization and autoclaving.

\section{MEMBRANE ELEMENTS AND MODULES}

Commercial ceramic membrane elements and modules vary in shape and size. Monoliths of channels (Figure 6) and tubes are the most common, but disks (or plates) are also available. The multichannel monolithic form of membrane elements offers the highest filtration packing density (filtration area per unit volume) among commercial ceramic membranes. It also has the advantages of higher mechanical strength and easier maintenance and repair. In the monolithic shape, the selective membrane layer usually is the innermost layer at the circumference of the channel while most of the solid portion of the element is the porous bulk support. Feed streams usually enter the channels at one end of the monolith and the retentate exits at the other end. In this crossflow configuration, the permeate passes through the membrane and the subsequent support layer(s). 


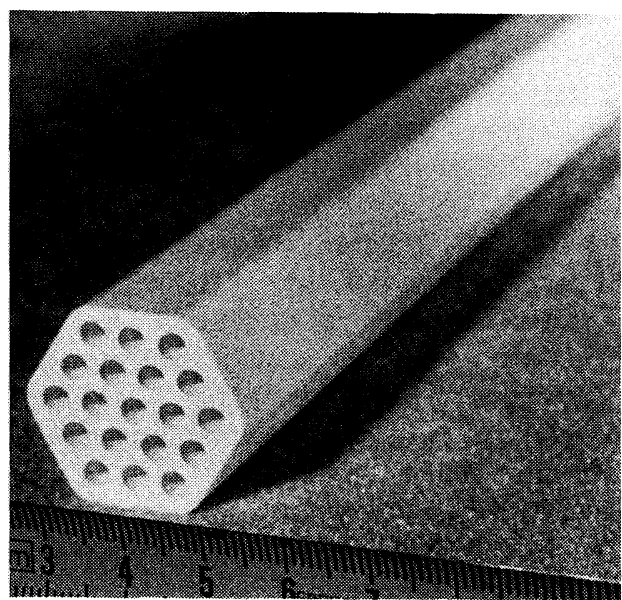

Figure 6. Photograph of a monolithic alumina membrane element containing 19 flow channels.

Monoliths and tubes can be further multiplied in a module to increase the filtration packing density. There have been developments on membrane end seals and membranemodule packing. Examples include cermet connections, graphite/carbon filaments, flexible graphite packing, localized cooling near the packing areas and compressible Knudsen flowtype seals. Matching of the thermal expansion coefficients between two materials in contact is one of the most important considerations to avoid cracking. In large-scale industrial applications, a ceramic membrane system may consist of several modules in parallel or in series or both.

\section{PREPARATION METHODS}

\section{Membranes with nearly straight pores}

Currently there is only one method for preparing commercial ceramic membranes with nearly straight and parallel pores (Figure 1). One side of a thin aluminum foil is oxidized anodically in an acid electrolyte. The unreacted portion is then etched with a strong acid to ensure open pores throughout the membrane thickness. These membranes can also be made into an asymmetric structure.

\section{Membranes with tortuous pores}

There are three major methods for preparing commercial ceramic membranes with tortuous pores: ceramic forming, slipcasting and phase separation/leaching.

Conventional ceramic forming techniques such as extrusion, compression and injection molding can be used to produce ceramic membranes with symmetric structures and large pores from particles of well controlled size distributions. In fact, most of the bulk supports used in multilayered ceramic membrane elements are made this way. Subsequent well-controlled drying and heat treatment are required to avoid shrinkage-induced cracking or pinholes.

In the slipcasting process, a stable dispersion of fine particles in a liquid medium (mostly water) is first prepared and brought into contact with a preformed dry porous support. Capillary force drives the liquid through the pores of the support, leaving a concentrated layer of particles superimposed on the support surface. Upon controlled 
drying and further heat treatments, the particle layer transforms from a semisolid to a wellbonded network of pores. Typically, this layer of membrane has a thickness of approximately $10 \mu \mathrm{m}$ or less to reduce pressure drop. The sizes of the dispersion particles relative to the support pore sizes are critical to avoid any appreciable pore blocking. Sometimes one or more intermediate layers may be required between the membrane and the bulk support.

An established way of making ceramic membranes with nanometer pore sizes by slipcasting is the sol-gel process in which the sol (dispersion of particles) is prepared by hydrolysis. The sol can then be transformed to gel by evaporating the dispersion medium from the sol. This sol-gel step and the subsequent heat treatment step can significantly influence the final membrane pore size. For example, Table I shows that the median pore diameters (by $\mathrm{N}_{2}$ desorption) of three alumina membranes prepared by a sol-gel process are affected by the water-to-aluminum sec-butoxide (ASB) and alcohol-to-ASB ratios used. The difference may be explained by the hydrolysis rate. With stoichiometrically excess water, the hydrolysis rate is high which yields colloidal suspension. When a minimum amount of water is used, inorganic-organic polymeric molecules are formed leading to an interconnected network. Table II shows the effect of the final calcination temperature on the pore size.

The third method of making ceramic membranes is to first induce phase separation by heating, for example, glass to form a water or acid leachable phase (sodium borate-rich) and a silica-rich phase. Upon leaching, the silica-rich phase remains and forms an isotropic and symmetric porous glass membrane (Figure 2).

\section{APPLICATIONS}

Almost all current large-scale commercial applications of ceramic membranes involve liquid phase. Many academic and industrial laboratories, however, are intensively studying gas phase applications at high temperatures. Two liquid phase applications will be reviewed in this paper: oil-water separation and protein separation. A gas phase application will be discussed where $\mathrm{H}_{2}$ and $\mathrm{N}_{2}$ are separated from a mixture. In all discussions, alumina membranes were used to obtain data.

\section{Oil-water separation}

When a relatively small quantity of oil or grease is present in water and needs to be removed or recovered, microporous ceramic membranes have been proven successful in many cases. While this may be applicable to the recovery of lubricants and other cases, the following discussion will address an application related to oil production.

Oil and grease are required to separate from the produced water in oil fields for overboard discharge and reinjection for waterflooding. Feed streams containing from 28 to $583 \mathrm{mg} / \mathrm{L}$ oil and grease and suspended and dissolved solids were processed at 32 to $65^{\circ} \mathrm{C}$ under an applied pressure difference of 0.35 to 2.76 atm with two 19-channel aluminamembrane monolithic modules in series. Membrane elements with pore diameters of 0.2 , 0.5 and $0.8 \mu \mathrm{m}$ were used in the modules. The crossflow velocity varied from 0.9 to 4.5 $\mathrm{m} / \mathrm{s}$. At a frequency of every $120 \mathrm{~s}$, a backflushing pressure of $5.4 \mathrm{~atm}$ for the duration of $0.5 \mathrm{~s}$ and a fastflushing velocity of $6 \mathrm{~m} / \mathrm{s}$ for a duration of $4-5 \mathrm{~s}$ were applied in combination with periodic chemical treatments (using strong caustic and strong nitric acid) to avoid fouling. The permeates passed through the membranes with a flux of 1445 to $2890 \mathrm{~L} / \mathrm{m}^{2}-\mathrm{h}$. The oil and grease concentrations of the feed, permeate and retentate samples shown in Table III clearly demonstrate the utility of alumina membranes for oil-water separation. 
TABLE I. Median pore diameters of unsupported alumina membranes ${ }^{a}$ affected by preparation conditions

\begin{tabular}{|c|c|c|c|}
\hline $\mathrm{m}\left(\mathrm{H}_{2} \mathrm{O}\right) / \mathrm{m}\left(\mathrm{ASB}^{\mathrm{b}}\right)$ & Alcohol & $\mathrm{m}(\mathrm{Alcohol}) / \mathrm{m}$ (ASB) & $\mathrm{d}(\mathrm{nm})$ \\
\hline 110 & - & 0 & 4.6 \\
\hline 3 & Propanol & 6 & 3.1 \\
\hline 3 & Butanol & 6 & 3.5 \\
\hline
\end{tabular}

a) Gels calcined at $425^{\circ} \mathrm{C}$.

b) Aluminum sec-butoxide.

TABLE II. Median pore diameters of unsupported alumina membranes ${ }^{a}$ affected by calcination temperatute ${ }^{\mathrm{b}}$

$\begin{array}{ll}\mathrm{T}\left({ }^{\circ} \mathrm{C}\right) & \mathrm{d}(\mathrm{nm}) \\ 425 & 35 \\ 600 & 50 \\ 800 & 55\end{array}$

a) Membranes prepared by hydrolysis of aluminum sec-butoxide at $110 \mathrm{~m}\left(\mathrm{H}_{2} \mathrm{O}\right) / \mathrm{m}(\mathrm{ASB})$.

b) Programmed heating to and holding at the final temperature for 1 hour.

TABLE III. Oil/grease concentrations ( $\mathrm{mg} / \mathrm{L}$ ) of feed, permeate and retentate samples of produced water

\begin{tabular}{cccc}
\hline Source of produced water & Feed & Permeate & Retentate \\
\hline 1 & $165-580$ & $<9^{\mathrm{a}}$ & $1265-3125$ \\
2 & $25-110$ & $<5^{\mathrm{a}}$ & $100-2900$ \\
\hline
\end{tabular}

a) Detection limit due to sample size.

\section{Protein separation}

Microporous ceramic membranes are preferred over organic membranes for some biotechnological applications such as protein separation and cell harvesting due to higher mechanical, chemical and thermal resistances. Clark et al. ${ }^{2}$ have recently studied crossflow ultrafiltration of a protein, bovine serum albumin (BSA), using multilayered alumina membrane tubes with pore diameters of 4,35 and $100 \mathrm{~nm}$. The inside diameter, outside diameter and the length of the membrane tubes are 7,10 and $750 \mathrm{~mm}$, respectively. Table IV shows the efficiency of the alumina membranes in separating the protein molecules. It was concluded that protein adsorption plays a significant role in the high retention of BSA by the 35 and $100 \mathrm{~nm}$ membranes. The permeate flux strongly depends on the $\mathrm{pH}$ of the solutions with the minimum occurring at $\mathrm{pH} 4.9$, the isoelectric point of $\mathrm{BSA}$.

\section{Other liquid phase applications}

Ceramic membranes have been successfully integrated into processing operations in food and beverage applications. Some specific examples are fruit juice clarification, removal of yeast or other microorganisms from beer while maintaining essential flavor, production of clean water and cheese making. 
TABLE IV. Retention coefficients of alumina membranes for separating bovine serum albumin (BSA)

\begin{tabular}{cccc}
\hline BSA conc. $\backslash$ Pore dia. & $4 \mathrm{~nm}$ & $35 \mathrm{~nm}$ & $100 \mathrm{~nm}$ \\
\hline $0.1 \mathrm{~g} / \mathrm{L}$ & 0.84 & 0.68 & 0.52 \\
$3.0 \mathrm{~g} / \mathrm{L}$ & $>0.98$ & $>0.98$ & $>0.98$ \\
\hline
\end{tabular}

a) Crossflow velocity of $8.4 \mathrm{~m} / \mathrm{s}$.

b) Tests at $32^{\circ} \mathrm{C}, \mathrm{pH} 6.6$ and 4 atm across membrane.

\section{Gas separation}

With their small pore sizes and inherent thermal stabilities, microporous ceramic membranes are ideal candidates for separating components in a gaseous mixture at high temperatures. It is often more economic to operate gas separation at the as-is elevated temperature of the feed gas, thus eliminating the cool-down/heat-up cycle required of organic membranes. The dominating gas transport mechanism(s) through the pores in the ceramic membranes depends on factors such as pore size, temperature, pressure and chemical nature of the gases involved. The pore diameters of current commercial ceramic membranes suitable for gas separation are in the range of a few hundred angstroms or less. Knudsen diffusion appears to be operative predominantly through those pores, particularly at high temperatures as shown in Figure 7 where the $\mathrm{CO}_{2}$ permeability at $450^{\circ} \mathrm{C}$ through the pores (mean pore diameter of $4 \mathrm{~nm}$ ) of an alumina membrane tube follows the Knudsen diffusion behavior while at $20^{\circ} \mathrm{C}$ the pronounced increase in the $\mathrm{CO}_{2}$ permeability above the Knudsen diffusion prediction is likely due to surface diffusion. In Figure 7 the prediction is based on a mean free path of 44.4 and $150.6 \mathrm{~nm}$ at 20 and $450^{\circ} \mathrm{C}$, respectively.

Given in Figure 8 are the comparison of experimental and calculated separation factors of $\mathrm{H}_{2}$ from $\mathrm{H}_{2}-\mathrm{N}_{2}$ binary mixtures at 20 and $538^{\circ} \mathrm{C}$. The calculated values are based on Knudsen diffusion. Within the conditions tested, experimental values are slightly less than the calculated ones. Although the separation factors for the $\mathrm{H}_{2}-\mathrm{N}_{2}$ gas mixtures are not very high, those for mixtures of $\mathrm{H}_{2}$ and hydrocarbon gases can be rather high. The reason is that, according to the Knudsen diffusion theory, the separation factor is inversely proportional to the square root of the ratio of the two molecular weights.

\section{Other gas phase applications}

Alumina membranes are currently used for removing particulates from clean room air. Large scale tests have been conducted in which ceramic membranes remove dust contaminants ( $>99 \%$ efficiency) from the gas stream entering gas turbines in coal-based electric power plants. Another area of gas phase applications being widely studied is membrane reactors at high temperatures. ${ }^{3}$ Most of the applications tested involve dehydrogenation or hydrogenation. When successfully implemented, ceramic membrane reactors can have profound impacts on improvements in product yield and selectivity and energy savings.

\section{CONCLUSIONS}

Microporous ceramic membranes having symmetric and asymmetric (multilayered) structures with nearly straight and tortuous pores are described in relation to their preparation methods which range from slipcasting, phase separation/leaching to anodic oxidation. Many of those ceramic membranes are mechanically, chemically and thermally stable, thus making them suitable for a wide spectrum of applications. Some representative applications are discussed: oil-water separation, protein separation and gas separation. Other applications holding great promises include food and beverage purification and concentration, gas filtration for clean rooms and power plants and membrane reactors. 


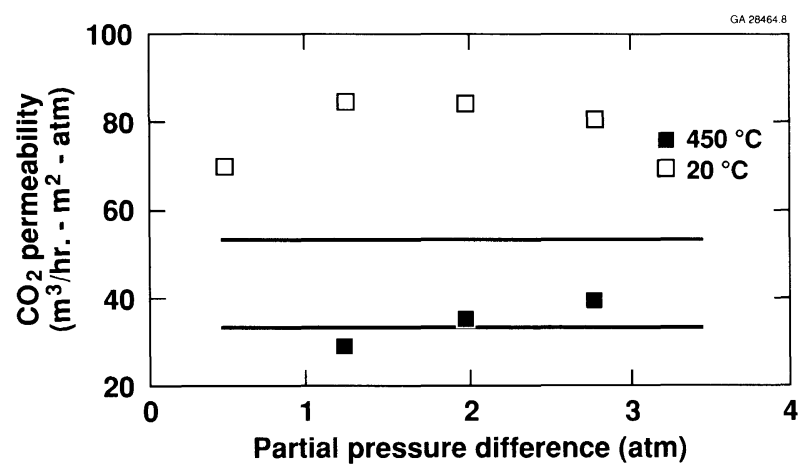

Figure 7. Carbon dioxide permeability of an alumina membrane with a median pore diameter of $4 \mathrm{~nm}$.

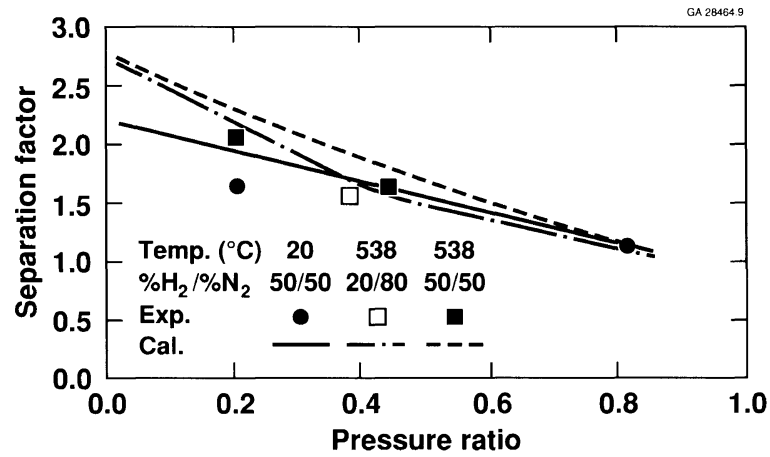

Figure 8. Separation factor of $\mathrm{H}_{2}$ from $\mathrm{H}_{2}-\mathrm{N}_{2}$ mixture (feed side pressure $=7 \mathrm{~atm}$ ).

\section{ACKNOWLEDGMENT}

Assistance of Drs. R. R. Bhave, A. S. C. Chen and G. Gallaher of Alcoa Separations Technology, Inc. and Prof. Y. H. Ma of Worcester Polytechnic Institute for providing some data used in this paper is appreciated.

\section{REFERENCES}

1. H. P. Hsieh, R. R. Bhave and H. L. Fleming, J. Membrane Sci., 39, 221 (1988).

2. W. M. Clark, A. Bansal, M. Sontakke and Y. H. Ma, J. Membrane Sci., in press (1990).

3. H. P. Hsieh, Catalysis Reviews - Sci. \& Eng., in press (1991). 